\title{
Análisis de la Formación de Hidrocarburos Aromáticos Policíclicos (PAH), sus derivados Oxigenados y Hollín en la Pirólisis de Compuestos Oxigenados usados como Aditivos a Combustibles
}

\author{
Fausto Viteri, Ángela Millera, Rafael Bilbao, Uxue Alzueta \\ Grupo de Procesos Termoquímicos (GPT) \\ Instituto de Investigación en Ingeniería de Aragón (I3A) \\ Universidad de Zaragoza, Mariano Esquillor s/n, 50018, Zaragoza, Spain. \\ Tel. +34-976762707, Fax +34-976762043, e-mail:fviteri@unizar.es
}

\begin{abstract}
Diferentes compuestos oxigenados se han propuesto como combustibles alternativos o aditivos a combustibles, debido a su capacidad de disminuir la emisión de algunos contaminantes atmosféricos y por su carácter renovable. La presente investigación se centra en el estudio de PAH, Oxy-PAH y hollín formados en la pirólisis de estos compuestos oxigenados.
\end{abstract}

\section{Introducción}

El constante uso de combustibles fósiles, para movilizar múltiples vehículos de transporte, hace que exista una mayor contaminación proveniente de este tipo de fuente. Existen varios compuestos responsables de la contaminación atmosférica que proceden de la quema de combustibles, entre los más importantes se encuentran el material particulado, formado principalmente por hollín, gases ligeros y otro grupo de gases complejos, los hidrocarburos aromáticos policíclicos (PAH). El hollín se forma dentro de las cámaras de combustión, en condiciones que incluyen altas temperaturas y bajas concentraciones de oxígeno. Los PAH son compuestos formados dentro de la cámara de combustión en zonas ricas de combustible, y se cree que este conjunto de compuestos constituye el primer paso para la formación de hollín. La United States Environmental Protection Agency (USEPA) ha clasificado 16 PAH, conocidos como 16 USEPA$\mathrm{PAH}$, como peligrosos para la salud por su carácter cancerígeno. Además, se ha observado que los derivados oxigenados de los PAH, los Oxy-PAH, tienen incluso mayores características tóxicas y mutagénicas [1].

Debido a la formación de distintos compuestos contaminantes en el proceso de combustión en motores, se han propuesto como biocombustibles algunos compuestos oxigenados o aditivos para combustibles de automoción. Diversas investigaciones han demostrado que varios compuestos oxigenados reducen la cantidad de contaminantes durante el proceso de combustión [2], como es el caso de las emisiones de material particulado, a un nivel muy bajo [3].

En la actualidad, se han utilizado ya varios compuestos oxigenados como biocombustibles o como aditivos a combustibles, como es el caso del etanol, el cual está siendo producido por varios países como Brasil [4].

El presente estudio busca investigar la formación de PAH, Oxy-PAH y hollín a partir de la pirólisis de compuestos oxigenados propuestos como biocombustibles o aditivos para combustibles.

\section{Objetivos y Metodología}

La investigación tiene como objetivos: i) Desarrollar un método de identificación y cuantificación de hidrocarburos aromáticos policíclicos oxigenados Oxy-PAH, ii) Conocer el proceso de formación de $\mathrm{PAH}$, Oxy-PAH y hollín durante la pirólisis de compuestos oxigenados de diferente grupo funcional, iii) Realizar análisis comparativos de la influencia de los compuestos estudiados sobre los productos formados.

El estudio se realiza utilizando instalaciones experimentales que constan de un sistema de alimentación de gases, un sistema de reacción y recolección de hollín, PAH y Oxy-PAH y un sistema de detección de gases ligeros.

El análisis de cuantificación de PAH utiliza el método desarrollado por nuestro grupo de investigación, utilizando cromatografía de gases acoplada a espectrometría de masas [5]. El procedimiento consiste en atrapar los $\mathrm{PAH}$ en dos 
fases: i) fase sólida, encontrada sobre la superficie del hollín y sobre las paredes del reactor, ii) fase gas, retenida por la resina XAD-2, la cual está empaquetada en un tubo delgado colocado detrás del sistema de recolección de hollín. Los PAH retenidos se someten a extracción Soxhlet y, luego, las muestras son concentradas utilizando un rotavapor y analizadas mediante el cromatógrafo de gases acoplado al espectrómetro de masas.

Todos los experimentos son llevados a cabo en un reactor de flujo de cuarzo, bajo condiciones de laboratorio bien controladas, en el intervalo de temperatura de 973-1473 K, usando diferentes concentraciones de entrada de los compuestos oxigenados considerados, entre 5000-50000 ppmv, y a presión atmosférica.

\section{Agradecimientos}

Los autores agradecen a MINECO y FEDER (Proyecto CTQ2012-34423) y al Gobierno de Aragón por la financiación recibida. Fausto Viteri agradece a la Universidad de Zaragoza y a la Secretaría Nacional de Educación Superior, Ciencia, Tecnología e Innovación (SENESCYT) de Ecuador, por la beca predoctoral otorgada.

\section{Referencias}

[1]. KARAVALAKIS, G., DEVES, G., FONTARAS, G., STOURNAS, S., SAMARAS, Z. and BAKEAS, E. The impact of soy-based biodiesel on PAH, nitro$\mathrm{PAH}$ and oxy-PAH emissions from a passenger car operated over regulated and nonregulated driving cycles. Fuel. 2010, 89(12), 3876-3883.

[2]. PEPIOT-DESJARDINS, P., PITSCH, H., MALHOTRA, R., KIRBY, S. and BOEHMAN, A. Structural group analysis for soot reduction tendency of oxygenated fuels. Combustion and Flame. 2008, 154(1-2),191-205.

[3]. WANG, J., WU, F., XIAO, J. and SHUAI, S. Oxygenated blend design and its effects on reducing diesel particulate emissions. Fuel. 2009, 88(10), 2037-2045.

[4]. CERQUEIRA LEITE, R., VERDE LEAL, M., BARBOSA CORTEZ, L., GRIFFIN, M. and GAYA SCANDIFFIO, M. Can Brazil replace 5\% of the 2025 gasoline world demand with ethanol? Energy. 2009, 34(5), 655-661.

[5]. SÁNCHEZ, N., SALAFRANCA, J., CALLEJAS, A., MILLERA, A., BILBAO, R. and ALZUETA, M. U. Quantification of polycyclic aromatic hydrocarbons (PAHs) found in gas and particle phases from pyrolytic processes using gas chromatography-mass spectrometry (GC-MS). Fuel. 2013, 107, 246-253. 